\title{
Successful treatment of cervical esophageal obstruction using combined antegrade and retrograde dilation with an endoscopic ultra- sound needle and fully covered stent
}

While complete esophageal obstruction is a technically challenging problem, the combined antegrade and retrograde dilation (CARD) procedure provides an endoscopic treatment option for these obstructions [1-3], and the addition of an endoscopic ultrasound (EUS) needle increases the speed and convenience of the procedure [4-5]. Here we describe use of this technique for the successful treatment of a long cervical esophageal occlusion by using flexible endoscopes and a fully covered removable stent.

A 61-year-old man presented to another institution with complete esophageal obstruction, 8 months after treatment for a T2N2B tonsillar squamous cell carcinoma. He required a gastrostomy tube for nutrition and was unable to swallow his own secretions. A conventional CARD procedure was attempted but unsuccessful due to the size of the obstruction.

Subsequently, the patient self-referred to our center for the CARD-EUS needle procedure. A 6-mm upper endoscope (GIFXP160; Olympus, Hamburg, Germany) was introduced through the gastrostomy and advanced retrograde to the distal aspect of the occlusion, which was located in the cervical esophagus ( $\bullet$ Fig. 1 a).

A 9-mm flexible upper endoscope (GIFQ180; Olympus) was advanced perorally to the proximal aspect of the stricture where C-arm fluoroscopy in the anteroposterior and lateral positions was used to align the endoscopes ( Fig. 1b). A 19guage EUS needle was advanced through the antegrade endoscope and used to traverse the $3.2-\mathrm{cm}$ obstruction under fluoroscopic guidance until visualized by the retrograde endoscope ( $\bullet$ Fig. 1c). A hydrophilic 0.035 -inch guide wire was then used to pass a 6-Fr biliary dilation catheter. Wire-guided balloon dilators were used to dilate the stricture to $11 \mathrm{~mm}$ at three stations with good effect $(\bullet$ Fig. 1 d,e). A $7-\mathrm{cm} \times 18-\mathrm{mm}$ fully covered removable stent was placed, taking care to
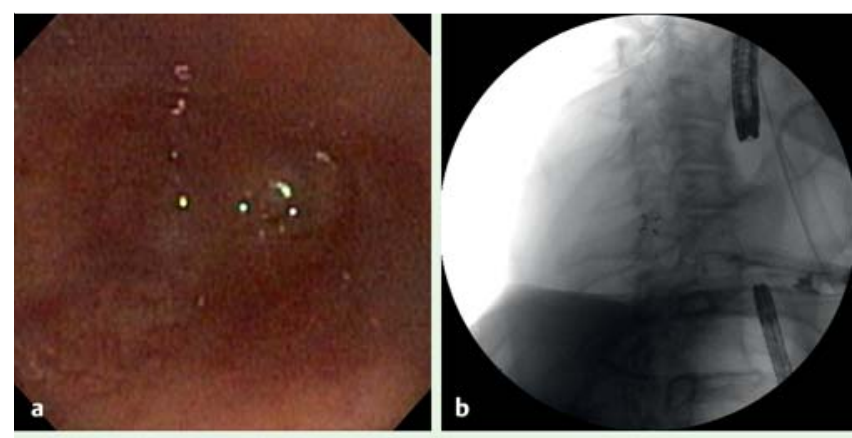

Fig. 1 a Original retrograde endoscopic view of the completely obstructed esophageal lumen. b Fluoroscopic images taken in anteroposterior and lateral views to assure correct scope alignment. c A 19-gauge endoscopic ultrasound (EUS) needle is advanced antegrade through the
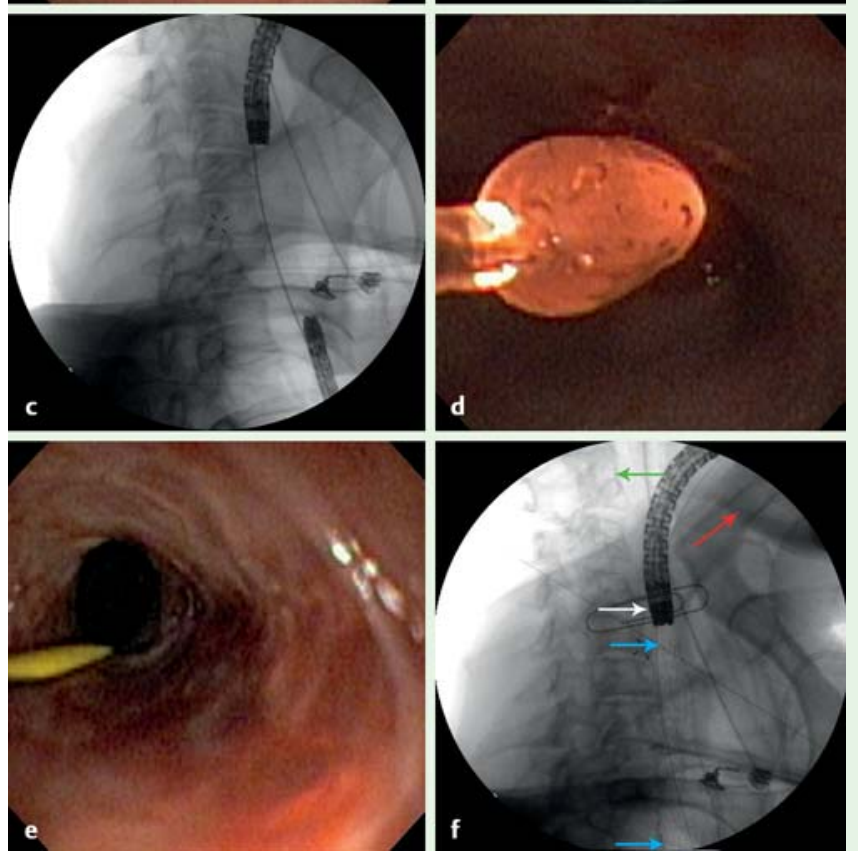
fibrotic obstruction under fluoroscopic guidance until it is visualized by the retrograde scope. d An 11-mm over-thewire balloon dilating the distal section of the esophageal obstruction, advanced and illuminated by the antegrade endoscope, as visualized by the retrograde endoscope. e Neo-esophageal lumen post dilation. f A removable, fully covered stent is placed to maintain patency under fluoroscopic guidance. Landmarks: jaw (red arrow), clavicle (green arrow), ends of deployed stent (blue arrows), esophageal inlet (white arrow).

open the proximal aspect of the occlusion without introducing the stent into the oropharynx, which proved difficult ( $\bullet$ Fig. 1f). The duration of the procedure was 80 minutes.

The stent was removed at 9 weeks, whereupon the patient was taught to perform periodic self-dilation with a Maloney rigid esophageal dilator, with good results.

Endoscopy_UCTN_Code_TTT_1AO_2AH

Competing interests: None
B. B. Ancrile, A. Mathew, M. T. Moyer Division of Gastroenterology and Hepatology, Penn State Milton S. Hershey Medical Center, Hershey, Pennsylvania, USA 


\section{References}

1 Baumgart DC, Veltzke-Schlieker W, Wiedenmann $B$ et al. Successful recanalization of a completely obliterated esophageal stricture by using an endoscopic rendezvous maneuver. Gastrointest Endosc 2005; 61: 473-475

2 Bueno R, Swanson SJ, Jaklitsch MT et al. Combined antegrade and retrograde dilation: a new endoscopic technique in the management of complex esophageal obstruction. Gastrointest Endosc 2001; 54: 368-372

3 Raju GS, Ahmed I. Team approach to the management of complex esophageal ob- struction. Gastrointest Endosc 2002; 55 304

4 Moyer MT, Stack BC Jr., Mathew A. Successful recovery of esophageal patency in 2 patients with complete obstruction by using combined antegrade retrograde dilation procedure, needle knife, and EUS needle. Gastrointest Endosc 2006; 64: 789-792

5 McGrath K, Brazer S. Combined antegrade and retrograde dilation: a new endoscopic technique in the management of complex esophageal obstruction. Gastrointest Endosc 2002; 56: $163-164$
Bibliography

DOI $10.1055 / \mathrm{s}-0030-1256084$

Endoscopy 2011; 43: E51 -E52

(c) Georg Thieme Verlag KG Stuttgart · New York . ISSN 0013-726X

\section{Corresponding author}

B. B. Ancrile, PhD

Division of Gastroenterology and Hepatology Penn State Milton S. Hershey Medical Center 500 University Drive

PO Box 850, Mail Code HU33

Hershey

Pennsylvania 17033-0850

USA

Fax: +1-717-531-6770

bancrile@hmc.psu.edu 